OPEN ACCESS

Edited by:

Takeshi Terao,

Oita University, Japan

Reviewed by:

Sam Cortese,

University of Southampton,

United Kingdom

Roumen Kirov,

Institute of Neurobiology

(BAS), Bulgaria

${ }^{*}$ Correspondence: Daryl Wayne Niedermoser daryl.niedermoser@upk.ch

Specialty section: This article was submitted to

Psychological Therapies,

a section of the journal

Frontiers in Psychiatry

Received: 31 October 2019 Accepted: 21 February 2020

Published: 17 March 2020

Citation:

Niedermoser DW, Kalak N, Kiyhankhadiv A, Brand S, Walter C, Schweinfurth N and Lang UE (2020)

Workplace-Related Interpersonal Group Psychotherapy to Improve Life

at Work in Individuals With Major

Depressive Disorders: A Randomized Interventional Pilot Study.

Front. Psychiatry 11:168. doi: 10.3389/fpsyt.2020.00168

\section{Workplace-Related Interpersonal Group Psychotherapy to Improve Life at Work in Individuals With Major Depressive Disorders: A Randomized Interventional Pilot Study}

\author{
Daryl Wayne Niedermoser ${ }^{1,2 *}$, Nadeem Kalak ${ }^{1}$, Anna Kiyhankhadiv ${ }^{1}$, Serge Brand ${ }^{1,3,4,5,6}$, \\ Corinna Walter ${ }^{1}$, Nina Schweinfurth ${ }^{1}$ and Undine E. Lang ${ }^{1}$ \\ 1 University Psychiatric Clinics (UPK), University of Basel, Basel, Switzerland, ${ }^{2}$ Departement of Business, Kalaidos University \\ of Applied Sciences, Zurich, Switzerland, ${ }^{3}$ Substance Abuse Prevention Research Center, Kermanshah University of Medical \\ Sciences, Kermanshah, Iran, ${ }^{4}$ Department of Sport, Exercise and Health, University of Basel, Basel, Switzerland, ${ }^{5}$ School of \\ Medicine, Tehran University of Medical Sciences, Tehran, Iran, ${ }^{6}$ Sleep Disorders Research Center, Kermanshah University of \\ Medical Sciences, Kermanshah, Iran
}

Objectives: Individuals suffering from major depressive disorder (MDD) often report workplace-related stress as the major cause of their disorder. Accordingly, workplacerelated stress was established as a fifth psychosocial problem area of Interpersonal Psychotherapy (workplace-related Interpersonal Psychotherapy, W-IPT). The aim of the study was to investigate the influence of W-IPT on depressive symptoms and on workplace-related issues in individuals with MDD compared to a treatment-as-usual (TAU) condition.

Methods: A total of 27 individuals with MDD (mean age $=43$ years, $48 \%$ males) were randomly assigned either to eight weekly group sessions of W-IPT or to the TAU condition. At baseline, 8 weeks later at the end of the intervention, and 20 weeks later at follow-up, the Hamilton Rating Scale for Depression was conducted. In addition, the participants completed the Beck Depression Inventory, the Work Ability Index (WAI), the Return to Work Attitude (RTW-SE), and the Insomnia Severity Index (ISI).

Results: Symptoms of depression in experts' ratings as well as in self-rated ratings decreased over time, but more so in the W-IPT condition compared to the TAU condition [experts rating: large effect size $(d=1.25)$ and self-assessment: large effect sizes $(d=$ 0.94)]. The subjective ability to work (WAl) [medium effect size $(d=0.68)$ ], self-efficacy to returning to work RTW-SE [medium effect size $(d=0.57)$ ], and subjective symptoms of insomnia (ISI) [large effect size $(d=1.15)]$ increased over time, but again more so in the $\mathrm{W}$ IPT condition compared to the TAU condition. The effects of the intervention remained stable from the end of the intervention to follow-up.

Conclusions: The pattern of results of this pilot study suggests that a newly established fifth IPT focus on workplace-related stress appeared to be particularly efficient in 


\section{individuals with MDD due to work-related stress in reducing depressive symptoms and reducing sleep complaints as well as in improving occupational outcomes.}

\section{Keywords: workplace-related stress, interpersonal psychotherapy, depression, ability to work, self-efficacy to returning to work, sleep}

\section{INTRODUCTION}

Major depressive disorder (MDD) is a common and disabling psychiatric disorder associated with an increase in disability and mortality (1). Based on the data using the disability-adjusted-lifeyears to assess "the sum years lost due to premature mortality and years lived with disability adjusted for severity," Murray and Lopez (2) estimated that MDD will be the third leading cause of burden worldwide by 2020. By contrast, Jorm et al. (3) claimed that the epidemiologic prevalence rates of MDD did not increase within the 30 years, while the social awareness of depression did.

Standard treatments of moderate and severe MDD consist mainly on the administration of antidepressants, i.e., serotoninreuptake inhibitors. More recent reviews and meta-analyses questioned the efficacy of antidepressants and the unpleasant side effects might be a reason why people with MDD often quit antidepressant medication (4-8). Other evidence-based treatment options are neuromodulation (9-11), cognitivebehavioral interventions $(12,13)$, or regular physical activity (14-17).

Treatment guidelines (AWMF, NICE) recommend antidepressants and/or psychotherapy to treat moderate and severe MDD. Individuals with major depressive disorders often report stressful issues related to the workplace. Job strain, low job control, low social support, high psychological demands, effortreward imbalance, and high job insecurity were confirmed as predictors particularly for depression (18-23). Several studies (24-27) show that, compared to healthy individuals, individuals with MDD are at an increased risk to lose their current job position $(24,25,27)$, to have more difficulties to go back to their workplace $(24,25,27)$, to find a new job, once they have been dismissed, and to keep their job position, when they return to their job after a period of illness-related unemployment. Furthermore, data on health costs show that, compared to an accident-related absence from work, depression-related absence from work cause higher economic burden for the individual (25, $27)$, the employer (27-29), and the public $(24,26,27,30)$. In addition, losing a job position or experiencing difficulties to go back to work turned out to be a risk factor for further relapses of MDD (24).

In this context, specific interventions to reduce workplacerelated stress in individuals with MDD might both reduce the economic costs for the public and the burden for the individual $(24,26,27,29,30)$.

Here we present the influence of the newly developed workplace-related Interpersonal Psychotherapy (W-IPT) which is a specific intervention to influence the workplace-related dimensions of symptoms of individuals with MDD. The original IPT concept was developed as a brief psychotherapeutic treatment aiming at the symptoms of an acute depressive disorder and current interpersonal problems and its effectiveness for the treatment of MDD has been widely demonstrated in numerous controlled trials [cf. meta-analysis from Cuijpers et al. (31)].

The W-IPT focuses on issues occurring in the context of the work role (32) such as social and interpersonal problems at work, mobbing, interpersonal conflicts, role transition, role confusion, burnout, boreout, job strain, low social support, effort-reward imbalance, job demand-control imbalance, and worklife imbalance.

The following three hypotheses and one research question were formulated and each of these is considered in turn. First, following Knekt et al. (33), Hange et al. (34), and Hallgren et al. (35), we expect that, compared to the TAU condition, the workplace-related dimensions of (a) the ability to work and (b) the self-efficacy in returning to work increased over time. The second hypothesis was in the context of empirical evidence (12, $31,36,37)$. We expect that the W-IPT treatment has positive effects on depressive symptoms when compared to the TAU condition. The symptoms of depression will improve in the experts' rating and in the self-rating. Following the second hypothesis and combining it with those of Santor and Kusumakar (38) and Lemmens et al. (37), we formulated the following exploratory research question. If there is a benefit in the W-IPT group, is the effect stable over a 3-month period measured at follow-up? Third, following Göder et al. (39) and Dombrovski et al. (40), we expected that, compared to the TAU condition, the W-IPT treatment will decrease the symptoms of insomnia.

\section{METHOD}

\section{Procedure}

We conducted a monocentric, randomized, controlled trial comparing W-IPT versus TAU condition in a group format in outpatients with major depression related to workplace-related issues between April 2018 and September 2019. The regional ethics commission (Ethikkommission Nordwest- und Zentralschweiz) approved the study (application number 201701-489). The eligible patients were fully informed about the study and signed the written informed consent at the first meeting. Moreover, all procedures were in line with the ethical standards of the Declaration of Helsinki (41) and its later amendments and with the ethical code of conduct of the American Psychological Association.

There was a total of one individual screening before the baseline and three data collections. The Hamilton Experts' Rating Scale for Depression (HRSD-24) was conducted in the screening part before the baseline as an information guide whether to include or exclude participants and to train (and 
explain) the questions. At baseline, the HRSD-24 was conducted and all questionnaires were filled out by the participants. The same procedure was applied at the last meeting ( 8 weeks after the start of the intervention) and at 12 weeks after the end of the treatment (follow-up at 20 weeks after randomization) (see Figure 1 and Table $\mathbf{1}$ ).

\section{Sample}

In-patients and out-patients were recruited through either a media announcement or a licensed practitioner. A total of 56 interested participants were clarified after a quick telephone/ e-mail screening. They were invited to an extended interview [interview, informed consent, Structured Clinical Interview for Disorders on the axis I (SCID-I), and HRSD-24]. A total of $28(50.0 \%)$ of the 56 patients fulfilled the inclusion criteria. The inclusion criteria were (1) being between 18 and 65 years of age, (2) major depressive disorder (SCID-I), (3) issues at or with the workplace (e.g., mobbing, effort-reward imbalance, and few social support), (4) fluent in German, (5) signed written informed consent, (6) complying with the study conditions, and (7) no or stable psychopharmacological and psychotherapeutic treatments 4 weeks before the study and throughout the study. The exclusion criteria were (1) HRSD$24<18$ ), (2) acute suicidality, (3) psychotic symptoms or bipolar disorder, (4) primary diagnosis of another mental illness, (5) substance use disorder, (6) personality disorder, and (7) applied (or already accepted) disability pension.

Of the 28 eligible participants, there was one non-starter and 27 started (mean age $=43$ years, $\mathrm{SD}=10.2,48 \%$ males) participating in the present study. There were 14 participants assigned to the W-IPT and 13 to the TAU condition (see Figure 1, CONSORT flow chart).

\section{Randomization}

A psychologist not otherwise involved in the study performed the randomization. A total of 15 blue and 15 red chips were put

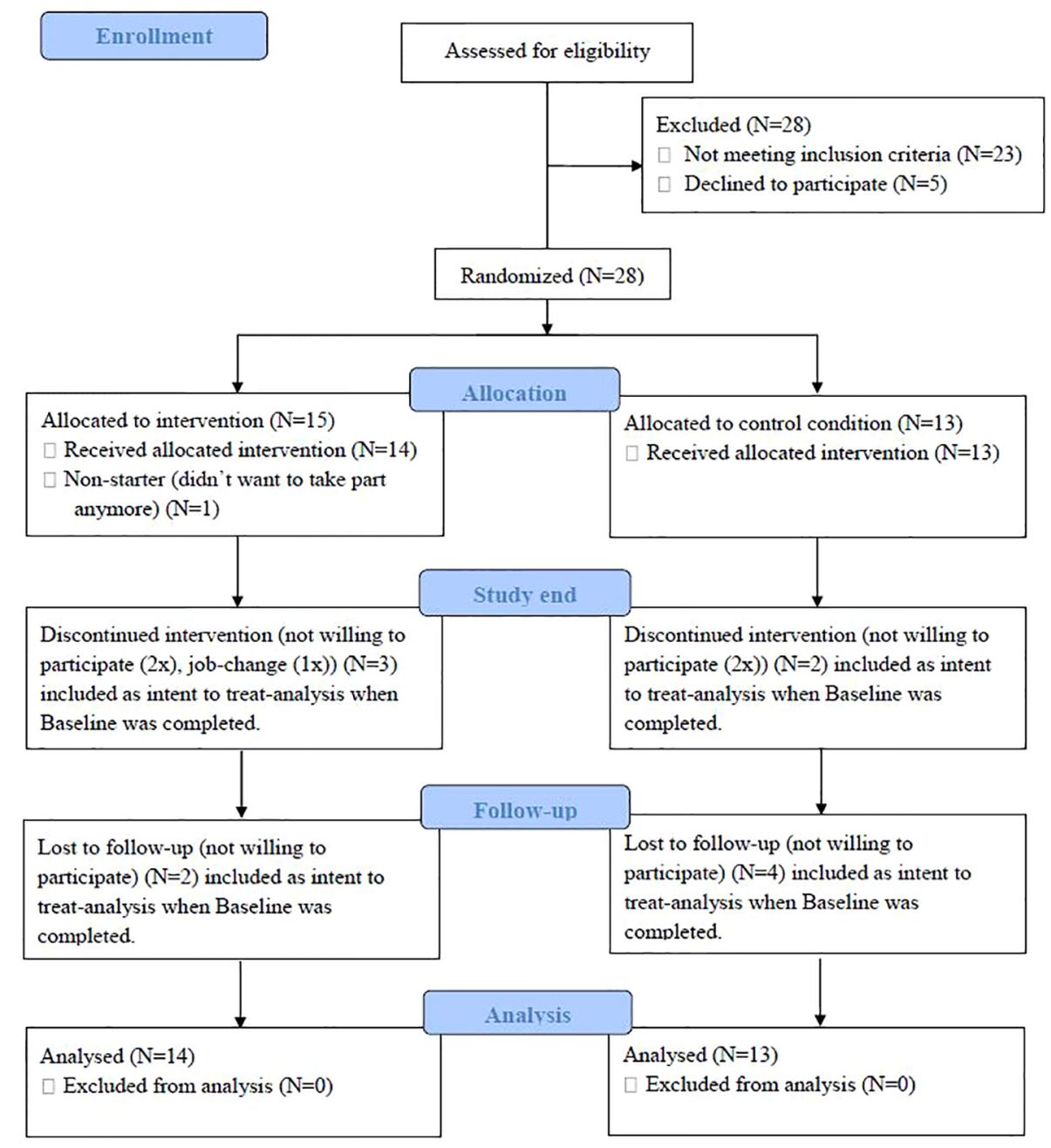

FIGURE 1 | CONSORT flow diagram. 
TABLE 1 | Overview of use and use of the questionnaires.

\begin{tabular}{lcccc}
\hline \multicolumn{1}{c}{ Questionnaires } & Prestart & Start & Study-end & Follow-up \\
\hline SCID-I & $X$ & & & \\
Informed Consent & $X$ & & & \\
Sociodemographics & $X$ & & & \\
Hamilton 24 & $X$ & $X$ & $X$ & $X$ \\
Becks Depression Inventory & & $X$ & $X$ & $X$ \\
Work Ability Index & & $X$ & $X$ & $X$ \\
Return to Work & & $X$ & $X$ & $X$ \\
Insomnia Severity Index & & $X$ & $X$ & $X$ \\
\hline
\end{tabular}

Prestart = initial consultation, SCID-I, Structured Clinical Interview DSM-IV.

in an opaque ballot box and stirred. The psychologist draws a chip and assigned the participant to the study condition. Then, the chip was put aside. To make sure that the blinding procedure is still clear, the participants were informed not to tell the psychologist anything about their treatment. After having taken part in the TAU condition, the participants were given the option to participate in a W-IPT group. The two groups did not differ as regards to age range, gender, civil status, employment, highest educational qualifications, antidepressants use, or in- and outpatient (see Table 2).

\section{Measurements}

To test our hypothesis, we used internationally recognized and well-established questionnaires in German. For several hypotheses, we used a few less-known questionnaires. These have been translated and translated back to ensure that the content is queried correctly. Mental disorders were assessed by means of the SCID-I based on DSM-IV $(42,43)$.

\section{Depression Questionnaires Experts' Rating}

The Hamilton Rating Scale for Depression (44) consisted of 24 items, asking on low mood, suicidality, irritability, tension, loss of appetite, loss of interests, and other somatic symptoms. Answers were given on rating scales differing from three-, four-, or five-point ratings. Higher sum scores reflect more marked depressive symptoms. Additionally, the scores were categorized as follows: 0-7, no depressive symptom/ remission; 8-17, mild; $18-24$, moderate; 25 and more, severe depressive disorder (Cronbach's alpha $=0.71$ ).

\section{Self-Rating}

The Beck's Depression Inventory (BDI-II) examines the depressive symptomatology from a self-perspective (45). The BDI-II is a widely established self-assessment tool with 21 items. It captures the affective, cognitive, and behavioral dimensions as well as the somatic symptoms of depression. The test quality criteria, such as internal consistency, validity, and test-retest reliability, are most satisfactory in both clinical and non-clinical subjects (46) (Cronbach's alpha $=0.89$ ).

\section{Workplace-Related Questionnaires}

The Work Ability Index (47) is a measurement tool that uses a questionnaire to calculate an index value for assessing the ability to work. This index value indicates the extent to which an employee is able to carry out his/her work on the basis of his/ her personal circumstances and existing working conditions $($ Cronbach's alpha $=0.70)$.

Return to Work Self-Efficacy (RTW-SE) is the belief in one's own ability to meet the demands required to return to work. The "return to work attitude" questionnaire (48) contains 11 questions on self-efficacy in managing demands at work (Cronbach's alpha $=0.66$ ).

\section{Subjective Sleep Quality Sleep Complaints Measured With the Insomnia Severity Index}

The questionnaire is in part in line with the DSM-IV insomnia criteria and measures current perception (within the last 2 weeks) in terms of symptom severity, stress, and impairment. These include severity of onset and persistence of sleep (midmorning and early morning wake disorder), satisfaction with the current sleep pattern, daily life impairment, occurrence of impairments due to sleep problem, and concern about sleep disorders [see Morin et al. (49) and Gerber et al. (50)] (Cronbach's alpha $=0.77$.

TABLE 2 | Sociodemographic and illness-related information, separately for participants in W-IPT and TAU condition.

\begin{tabular}{|c|c|c|c|}
\hline & \multicolumn{2}{|c|}{ Groups } & \multirow[t]{2}{*}{ Statistics } \\
\hline & W-IPT & TAU & \\
\hline $\mathrm{N}$ & 14 & 13 & \\
\hline Age range in years & $24-55$ & $28-55$ & \\
\hline Age in years $M(S D)$ & $40.86(11.29)$ & $45.77(8.438)$ & $t(25)=-1.279, p=.213$ \\
\hline Gender (female/male) & $7 / 7$ & $7 / 6$ & $X^{2}(N=27 d f=1)=0.04, p=.84$ \\
\hline Civil status (single/married) & $10 / 4$ & $9 / 4$ & $X^{2}(N=27, d f=1)=0.16, p=.90$ \\
\hline Current job position (yes/no) & $11 / 3$ & $9 / 4$ & $X^{2}(N=27, d f=1)=0.31, p=.58$ \\
\hline Educational level (high school/diploma/university degree) & $1 / 6 / 7$ & $1 / 5 / 7$ & $X^{2}(N=27, d f=2)=0.05, p=.97$ \\
\hline Antidepressants (yes/no) & $10 / 4$ & $7 / 6$ & $X^{2}(N=27, d f=1)=1.82, p=.18$ \\
\hline Participants (In-/Outpatient) & $7 / 7$ & $4 / 9$ & $X^{2}(N=27, d f=1)=0.93, p=.34$ \\
\hline
\end{tabular}




\section{Intervention}

\section{Work-Related Interpersonal Psychotherapy}

The W-IPT condition followed a guideline (51, manuscript in preparation). W-IPT focuses on the work context by adding specific elements to the regular IPT strategies: identifying work-related stress factors using an individual stress and resource profile, creating a balance between performance values and interpersonal values, teaching mindfulness and coping strategies for work-related stress (including social support), and practicing social communication skills at work to cope with conflict and role changes. By applying those strategies, we address social and interpersonal problems at work, such as mobbing, interpersonal conflicts, role transition, role confusion, burnout, boreout, job strain, low social support, and work-life imbalance (for more details, see Table 3). The intervention includes one weekly group session of $90 \mathrm{~min}$ for six to eighr participants over 8 weeks in addition to the TAU condition. Trained psychotherapists, certified in IPT treatment, conducted the group sessions.

\section{TAU Condition}

The participants randomized to the TAU condition received no further intervention during the study period and were encouraged to continue with attending appointments with their clinicians. In order to compensate for non-participation in the W-IPT condition, the patients in the TAU condition were offered to participate in the W-IPT program after their followup evaluation.

\section{Statistical Analysis}

Following Julious (52), a caseload of 12 individuals per group in pilot studies is reasonable to start with. A series of t-tests and Pearson's correlations was performed to compare sociodemographic, workplace-related, depression-related, and sleep complaints information between the two groups.

To assess changes over time and between and within the two groups, a series of ANOVAs for repeated measures was performed with the factors time (start, study end, and followup), group (intervention and TAU), and the time by group interaction and with symptoms of depression (experts' ratings and self-ratings), work-related information, and subjective sleep complaints as dependent variables. Due to the deviation of sphericity, the ANOVAs were performed using Greenhouse-Geisser corrected degrees of freedom, although the original degrees of freedom are reported with the relevant Greenhouse-Geisser epsilon value $(\epsilon)$. All computations were performed with the intention-to-treat analysis, with the last observation carried forward method. For ANOVAs, the effect sizes were reported as partial eta squared $\left(\eta_{\mathrm{p}}{ }^{2}\right)$, with $0.01<\eta_{\mathrm{p}}{ }^{2}<0.059$ indicating small (S), $0.06<\eta_{\mathrm{p}}{ }^{2}<0.139$ indicating medium $(\mathrm{M})$, and $\eta_{\mathrm{p}}{ }^{2}>0.14$ indicating large $(\mathrm{L})$ effect sizes. In addition, we followed Becker (53) and reported effect sizes for t-tests within and between the groups for all time points. To classify the effect sizes, we followed Cohen (54): effect sizes can be evaluated as trivial ( $\mathrm{T}$; $d=0-0.19$ ), small ( $\mathrm{S} ; d=0.20-0.49)$, medium ( $\mathrm{M} ; d=0.50-0.79)$, or large ( $\mathrm{L} ; d=0.80$ and greater). The level of significance was set at alpha $p \leq .05$. All statistical calculations were performed with SPSS $^{\circledR} 25.0$ (IBM Corporation, Armonk, NY, USA) for Windows ${ }^{\circledR}$.

\section{RESULTS}

All descriptive, inferential, and statistical indices are reported in Tables 4-6, and Figure 2. Table 4 shows the descriptive statistics, Table 5 shows the inference statistics, Table $\mathbf{6}$ shows the effect size comparisons, and Figure 2 shows the work ability index.

\section{Symptoms of Depression: Experts' Ratings}

Experts' rated symptoms of depression decreased over time (large effect size), but more so in the W-IPT group compared to the TAU condition (medium effect size). Experts' rated symptoms of depression was lower in the W-IPT group (medium effect size). Within the W-IPT group, the symptoms of depression decreased from baseline to the end

TABLE 3 | Content of the sessions on workplace-related interpersonal psychotherapy.

\begin{tabular}{|c|c|}
\hline Sessions & Content of sessions \\
\hline One & $\begin{array}{l}\text { Introducing members, identify work-related stress and dysbalances in the context of depression, theory and information, mindfulness exercise, } \\
\text { providing handouts and homework }\end{array}$ \\
\hline Two & $\begin{array}{l}\text { Reflecting and discussing the homework, thoughts and feelings, introduction to self-care and mindfulness, role play exercise, and quick meditation, } \\
\text { providing handouts and homework }\end{array}$ \\
\hline Three & $\begin{array}{l}\text { Reviewing homework, interpersonal stress and conflicts, introducing the Kiesler circle model, role play exercise, do not fall victim, mindfulness } \\
\text { meditation, providing handouts and homework }\end{array}$ \\
\hline Four & $\begin{array}{l}\text { Staying in the present, mindfulness exercise of the body scan, communication at the workplace, how to solve conflicts at the workplace (using the } \\
\text { Kiesler model), discussing the homework, providing handouts and homework }\end{array}$ \\
\hline Five & $\begin{array}{l}\text { Reflecting and discussing the homework, define and identify the living values, handout and discussion of what's important to me, building a } \\
\text { supportive network, work-life balance, self-compassion exercise, providing handouts and homework }\end{array}$ \\
\hline Six & Mindfulness exercise, reviewing the homework, role play, to ask for help, providing handouts and homework \\
\hline Seven & Mindfulness exercise, implementation of the values at the workplace, change and acceptance, providing handouts and homework \\
\hline Eight & $\begin{array}{l}\text { Reviewing and discussion of the homework, balance between acceptance and change, applying what was learned, conducting a survey of the } \\
\text { course, conclusion and finishing the course along with last contemplation }\end{array}$ \\
\hline
\end{tabular}


TABLE 4 | Descriptive overview of the descriptive statistics, separately for assessment time (start, study-end, and follow-up) and group (W-IPT vs. TAU condition).

\begin{tabular}{|c|c|c|c|c|c|c|}
\hline & \multicolumn{6}{|c|}{ Assessment times } \\
\hline & \multicolumn{2}{|c|}{ Baseline } & \multicolumn{2}{|c|}{ Study-end (+ 8 weeks) } & \multicolumn{2}{|c|}{ Follow-up (Total of 20 weeks) } \\
\hline & $\begin{array}{c}\text { W-IPT (N=14) } \\
\text { M (SD) }\end{array}$ & $\begin{array}{c}\text { TAU }(N=13) \\
M(S D)\end{array}$ & $\begin{array}{l}\text { W-IPT } \\
M \text { (SD) }\end{array}$ & $\begin{array}{c}\text { TAU } \\
\text { M (SD) }\end{array}$ & $\begin{array}{l}\text { W-IPT } \\
\text { M (SD) }\end{array}$ & $\begin{array}{c}\text { TAU } \\
M \text { (SD) }\end{array}$ \\
\hline Hamilton 24 & $25.5(4.88)$ & $27.46(12.18)$ & $16.36(9.16)$ & $23.85(15.88)$ & $16.29(10.62)$ & $22.39(14.38)$ \\
\hline Becks Depression Inventory & $24.07(8.11)$ & $28.23(12.23)$ & $16.14(8.74)$ & $21.92(13.81)$ & $15.71(10.74)$ & $20.85(12.50)$ \\
\hline Work Ability Index & $27.14(10.48)$ & $26.58(7.80)$ & $30.64(10.12)$ & $28.08(10.22)$ & $33.75(9.58)$ & $27.54(9.50)$ \\
\hline Return to Work & $28.21(5.22)$ & $27.62(6.23)$ & $31(4.56)$ & $27(6.30)$ & $31.79(5.45)$ & $28.15(6.89)$ \\
\hline Insomnia Severity Index & $14(5.19)$ & $14.67(5.71)$ & $8.64(4.11)$ & $13.31(7.25)$ & $7.79(5.37)$ & $12.23(7.11)$ \\
\hline
\end{tabular}

TABLE 5 | Inferential statistics of depression, work, and sleep, with the factors Time (start, study-end \& follow-up), Group (intervention vs. TAU), and the Time $\times$ Group interaction.

\begin{tabular}{|c|c|c|c|c|c|c|c|}
\hline & \multicolumn{7}{|c|}{ Factors } \\
\hline & \multicolumn{2}{|c|}{ Time } & \multicolumn{2}{|c|}{ Group } & \multicolumn{2}{|c|}{ Time $\times$ Group } & \multirow{2}{*}{$\frac{\text { Greenhouse-Geisse }}{\text { Epsilon }}$} \\
\hline & $F$ & $\eta_{p}^{2}$ & $\boldsymbol{F}$ & $\eta_{p}^{2}$ & $\boldsymbol{F}$ & $\eta_{p}^{2}$ & \\
\hline Hamilton 24 & $12.048^{\star \star \star}$ & $0.325[\mathrm{~L}]$ & 1.610 & $0.060[\mathrm{M}]$ & 1.618 & $0.061[\mathrm{M}]$ & .995 \\
\hline Becks Depression Inventory & $15.083^{\star \star \star}$ & $0.376[\mathrm{~L}]$ & 1.674 & $0.063[\mathrm{M}]$ & 0.133 & $0.005[S]$ & .854 \\
\hline Work Ability Index & $3.904^{*}$ & $0.135[\mathrm{M}]$ & $4.981^{*}$ & $0.166[\mathrm{~L}]$ & 2.159 & $0.079[\mathrm{M}]$ & .976 \\
\hline Return to Work & 3.120 & $0.111[\mathrm{M}]$ & 1.843 & $0.069[\mathrm{M}]$ & 2.571 & $0.093[\mathrm{M}]$ & .955 \\
\hline Insomnia Severity Index & $12.616^{\star \star \star}$ & $0.335[\mathrm{~L}]$ & 2.676 & $0.097[\mathrm{M}]$ & 3.032 & $0.108[\mathrm{M}]$ & .706 \\
\hline
\end{tabular}

Degrees offreedom - Time: $(2,25)$, Group: $(1,25)$, Time $\times$ Group $(1,25) . S$, small effect size; $M$, medium effect size; $L$, large effect size. $S>0.01, M>0.06, L<0.14 .{ }^{*} p<0.05 ;{ }^{* * *} p<0.001$.

TABLE 6 | Effect sizes for mean comparisons form pre- to post-assessment within the groups (intervention group and TAU group).

\begin{tabular}{|c|c|c|c|c|c|c|}
\hline \multirow[t]{3}{*}{ Group } & \multicolumn{2}{|c|}{ Start to study-end } & \multicolumn{2}{|c|}{ Start to follow-up } & \multicolumn{2}{|c|}{ Study-end to follow-up } \\
\hline & W-IPT & TAU & W-IPT & TAU & W-IPT & TAU \\
\hline & Cohen's d & Cohen's d & Cohen's d & Cohen's d & Cohen's d & Cohen's d \\
\hline Hamilton 24 & $1.246[\mathrm{~L}]$ & $0.255[S]$ & 1.115 [L] & $0.381[S]$ & 0.007 [1] & $0.096[1]$ \\
\hline Becks Depression Inventory & $0.940[\mathrm{~L}]$ & $0.484[S]$ & $0.878[\mathrm{~L}]$ & $0.597[\mathrm{M}]$ & $0.044[1]$ & $0.082[1]$ \\
\hline Work Ability Index & $0.680[\mathrm{M}]$ & $0.165[S]$ & $0.658[\mathrm{M}]$ & $0.111[1]$ & 0.315 [S] & 0.055 [1] \\
\hline Return to Work & $0.569[\mathrm{M}]$ & 0.099 [S] & $0.671[\mathrm{M}]$ & $0.081[1]$ & 0.157 [1] & $0.174[1]$ \\
\hline Insomnia Severity Index & $1.145[\mathrm{~L}]$ & $0.212[S]$ & $1.177[\mathrm{~L}]$ & $0.382[\mathrm{~S}]$ & $0.179[1]$ & $0.150[1]$ \\
\hline
\end{tabular}

$T$, trivial effect size; $S$, small effect size; $M$, medium effect size; $L$, large effect size.

of the study (large effect size) and from baseline to follow-up (large effect size); from the end of the study to follow-up, the symptoms of depression remained stably low (trivial effect size). Within the TAU group, the symptoms of depression decreased from baseline to the end of the study (small effect size) and from baseline to follow-up (small effect size); from the end of the study to follow-up, the symptoms of depression remained stably low (trivial effect size).

\section{Symptoms of Depression: Self-Ratings}

The self-rated symptoms of depression decreased in both groups over time (large effect size), but in the W-IPT group, the symptoms of depression decreased more compared to those of the TAU condition (small effect size). The self-rated symptoms of depression were lower in the W-IPT group (medium effect size). Within the W-IPT group, the symptoms of depression decreased from baseline to the end of the study (large effect size) and from baseline to follow-up (large effect size); from the end of the study to follow-up, the symptoms of depression remained stably low (trivial effect size). Within the TAU group, the symptoms of depression decreased slightly from baseline to the end of the study (small effect size) and from baseline to follow-up (medium effect size); from the end of the study to follow-up, the symptoms of depression remained stably high (trivial effect size). 


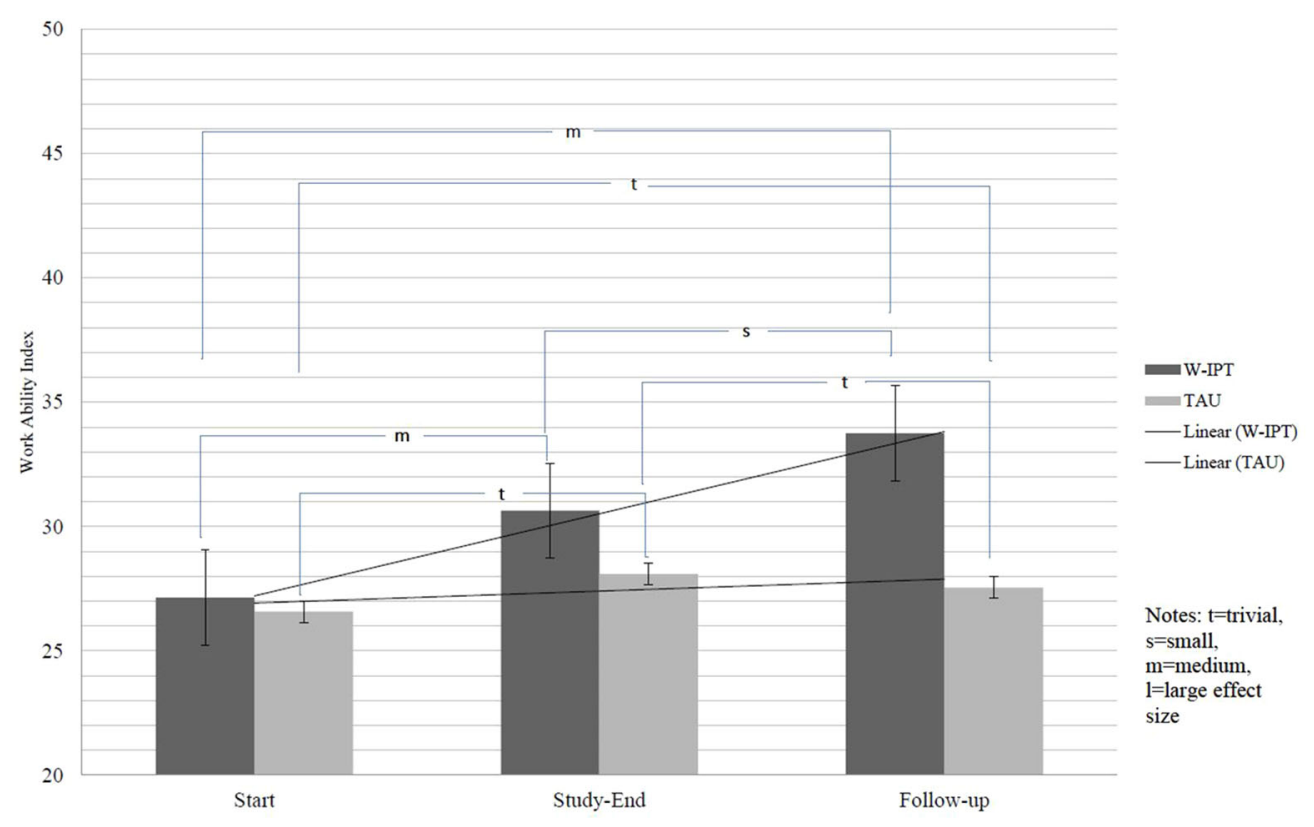

FIGURE 2 | Work Ability Index (WAI).

\section{Ability to Work: Self-Ratings}

The self-rated ability to work increased in both groups over time (medium effect size), but in the W-IPT group, the participants reported a higher self-rated ability to work compared to the TAU condition (medium effect size). The self-rated ability to work was higher in the W-IPT group (large effect size). Within the W-IPT group, the self-rated ability to work increased from baseline to the end of the study (medium effect size) and from baseline to follow-up (medium effect size); from the end of the study to follow-up, the ability to work remained stably low (small effect size). Within the TAU group, the self-rated ability to work increased slightly from baseline to the end of the study (small effect size) and from baseline to follow-up (trivial effect size); from the end of the study to follow-up, the ability to work remained stably low (trivial effect size) (cf. Figure 2).

\section{Return to Work: Self-Efficacy, Self-Ratings}

The self-rated return to work increased in both groups over time (medium effect size), but in the W-IPT group, the selfrated return to work self-efficacy increased more compared to the TAU condition (medium effect size). The self-rated return to work was higher in the W-IPT group (medium effect size). Within the W-IPT group, the self-rated return to work increased from baseline to the end of the study (medium effect size) and from baseline to follow-up (medium effect size); from the end of the study to follow-up, return to work remained stably high (trivial effect size). Within the TAU group, the self-rated return to work increased slightly from baseline to the end of the study (small effect size) and from baseline to follow-up (trivial effect size); from the end of the study to follow-up, return to work remained stably low (trivial effect size).

\section{Subjective Sleep Quality} Insomnia Severity Index: Self-Ratings

The self-rated sleep complaints decreased in both groups over time (large effect size), but more so in the W-IPT group compared to the TAU condition (medium effect size). The self-rated sleep complaints were lower in the W-IPT group (medium effect size). Within the W-IPT group, the self-rated sleep complaints decreased from baseline to the end of the study (large effect size) and from baseline to follow-up (large effect size); from the end of the study to follow-up, the selfrated sleep complaints remained stably low (trivial effect size). Within the TAU group, the self-rated sleep complaints decreased only slightly from baseline to the end of the study (small effect size) and from baseline to follow-up (small effect size); from the end of the study to follow-up, the self-rated sleep complaints remained stably high (trivial effect size)

To summarize, the symptoms of depression (experts' rating) decreased in both groups over time (large effect sizes), but in the W-IPT group, the symptoms of depression decreased more compared to those of the TAU group. The reduction at follow-up led the $\mathrm{W}$-IPT group to an altered assessment of the severity of the depression diagnosis (from moderate to mild), whereas in the TAU condition the 
reduction did not lead to a change in the assessment. The symptoms of depression (self-rating) decreased in both groups over time (large effect sizes), but in the W-IPT, the symptoms of depression decreased more compared to those of the TAU group. The work ability index self-rated interpretation gained in both groups over time (medium effect size), but in the W-IPT, the work ability increased more compared to that of the TAU group. The self-efficacy for the return to work gained in both groups over time (medium effect size), but in the W-IPT, the self-efficacy increased more compared to that of the TAU group. The self-rated symptoms of subjective sleep complaints decreased in both groups over time (large effect size), but in the W-IPT group, the sleep complaints decreased more compared to those of the TAU.

\section{DISCUSSION}

The key findings of the present study suggest that a specific work-related approach for depressed patients with work stress led to a greater decrease of clinician- and self-rated depressive symptoms compared to TAU. In addition, not only the subjective work ability was higher and the self-efficacy was more improved in the W-IPT group but also the quality of sleep was more increased compared to the TAU condition. Importantly, those effects remained stable over the follow-up time period. The particularly large effect in the W-IPT condition indicates that a job-related focus can help in the short and long terms.

The present study adds to the current literature in an important way because not only is the IPT beneficial for individuals with MDD $(31,55)$ but also it is especially based on psychosocial and workplace-related issues [see also Schramm and Berger (32)]. Our main study strength is the value of longitudinal research for extending knowledge about the relationship between W-IPT and MDD because of workplace-related stress.

The results are important for clinicians because the newest meta-analysis (8) showed that it is unclear whether the antidepressive treatment is more efficacious than the placebo treatment. There is an obvious necessity to use best evidencebased practices. The psychotherapeutic treatments are well evaluated, especially the cognitive-behavioral aspect, and IPT seems to be the preferred choice. The present study results are in line with the results shown before.

To summarize, all of our hypotheses plus the exploratory research question were fully confirmed. In this respect, comparing the effect of the intervention with those of the previous studies (see "Introduction"), again we hold that the present finding is clinically relevant. Thus, the present finding is in accord with previous results (33-35). We believe that the beneficial influence of W-IPT cannot be explained merely as an effect of time as they outperformed the outcome of the TAU condition.

In terms of comparing the long-term effect sizes of the present study with those of the previous studies, the present finding is in accord with previous results $(37,38)$.
We expected the work ability in the intervention group to be higher than in the TAU group. Surprisingly, the work ability in the W-IPT condition grew further even after the end of the study and over the follow-up time from originally poor to medium at the end of the intervention and almost good at follow-up, while the TAU condition showed a stable trend of poor work ability.

Despite the intriguing findings, several limitations warrant against overgeneralization of the present results. First, the sample size was small, although we basically relied on effect size calculations which, unlike $p$-values, do not vary with sample size, but since this was a pilot study, the small numbers can be explained. Further, there are increasing concerns about the importance and 'significance' of $p$-values (56). Second, the sample with a broad age range can be seen not only as a possible limitation but also as a strong point. After all, there was a positive influence despite the heterogeneity of the sample.

Further studies might differentiate between specific groups (f.i., gender, age, or comorbidities). Another limitation is the relative short follow-up period of 3 months. A longer follow-up could be helpful, especially in practical uses (employee training). Booster sessions could be provided to ensure sustainable effects. In addition, our study compared the possible effect of a new group program with a TAU condition. We cannot definitively say whether or not it is superior to other evidence-supported psychological treatments such as cognitive behavioral therapy. Furthermore, the inclusion and exclusion criteria were chosen to identify a particular kind of sample. However, comorbidities such as substance use or personality disorders are common in depressive disorders. Lastly, it is possible that further latent but unassessed variables (family members, sleep-quality interaction, and increase in social support) might have biased the results shown (in any given direction possible).

\section{CONCLUSIONS}

An 8-week group intervention with W-IPT had improved symptoms of depression, ability to work, self-efficacy regarding return to work, and sleep complaints more than the TAU condition.

We believe that the present results might be of broader interest: First, in terms of the costs for the companies (hiring new employee and absent or less productive employees) and for the employees (health and changing the job). Specifically, costs in employees being less productive or absent will result in several high costs per employee $(26,27,30,57)$. Second, the public health cost should also be mentioned here although we did not calculate the costs. Third, it follows that the benefit of an early investment in individual health should be the main interest of all three partners.

A short efficient treatment in groups seems to be easy to implement and economical. It could be a fixed part of a regular occupational health management $(58,59)$. The possible uses are (1) companies interested in having a modern occupational health management and (2) individuals experiencing stress and problems at the workplace for secondary or tertiary prevention. 


\section{DATA AVAILABILITY STATEMENT}

The raw data supporting the conclusions of this article will be made available by the authors, without undue reservations, to any qualified researchers.

\section{ETHICS STATEMENT}

The studies involving human participants were reviewed and approved by Ethikkommission Nordwest- und Zentralschweiz (EKNZ)-application number 2017-01-489. The patients/ participants provided their written informed consent to participate in this study.

\section{AUTHOR CONTRIBUTIONS}

$\mathrm{DN}, \mathrm{NK}, \mathrm{AK}, \mathrm{SB}, \mathrm{CW}, \mathrm{NS}$, and UL wrote the proposal and designed the study and completed the final draft. DN, NK, AK, $\mathrm{SB}, \mathrm{CW}$, and NS were involved in data gathering and data

\section{REFERENCES}

1. American Psychiatric Association. Diagnostic and Statistical Manual of Mental Disorders 5th edition: DSM 5. Arlington VA: American Psychiatric Association (2013).

2. Murray CJ, Lopez AD. Global mortality, disability, and the contribution of risk factors: Global Burden of Disease Study. Lancet (1997) 349:1436-42. doi: 10.1016/S0140-6736(96)07495-8

3. Jorm AF, Patten SB, Brugha TS, Mojtabai R. Has increased provision of treatment reduced the prevalence of common mental disorders? Review of the evidence from four countries. World Psychiatry (2017) 16:90-9. doi: 10.1002/ wps.20388

4. Boesen K, Paludan-Muller AS, Munkholm K. Network meta-analysis of antidepressants. Lancet (2018) 392:1011. doi: 10.1016/S0140-6736(18) 31783-5

5. Hengartner MP, Ploderl M. Statistically Significant Antidepressant-Placebo Differences on Subjective Symptom-Rating Scales Do Not Prove That the Drugs Work: Effect Size and Method Bias Matter! Front Psychiatry (2018) 9:517. doi: 10.3389/fpsyt.2018.00517

6. Hengartner MP. Scientific debate instead of beef; challenging misleading arguments about the efficacy of antidepressants. Acta Neuropsychiatr (2019) 31:235-6. doi: 10.1017/neu.2019.23

7. Hengartner MP, Ploderl M. Newer-Generation Antidepressants and Suicide Risk in Randomized Controlled Trials: A Re-Analysis of the FDA Database. Psychother Psychosom (2019) 88:1-2. doi: 10.1159/000501215

8. Munkholm K, Paludan-Muller AS, Boesen K. Considering the methodological limitations in the evidence base of antidepressants for depression: a reanalysis of a network meta-analysis. BMJ Open (2019) 9:e024886. doi: 10.1136/ bmjopen-2018-024886

9. Gaynes BN, Lloyd SW, Lux L, Gartlehner G, Hansen RA, Brode S, et al. Repetitive transcranial magnetic stimulation for treatment-resistant depression: a systematic review and meta-analysis. J Clin Psychiatry (2014) 75:477-89. quiz 489. doi: 10.4088/JCP.13r08815

10. Ren J, Li H, Palaniyappan L, Liu H, Wang J, Li C, et al. Repetitive transcranial magnetic stimulation versus electroconvulsive therapy for major depression: a systematic review and meta-analysis. Prog Neuropsychopharmacol Biol Psychiatry (2014) 51:181-9. doi: 10.1016/j.pnpbp.2014.02.004

11. Salehi I, Hosseini SM, Haghighi M, Jahangard L, Bajoghli H, Gerber M, et al. Electroconvulsive therapy (ECT) and aerobic exercise training (AET) increased plasma BDNF and ameliorated depressive symptoms in patients entering. DN, NK, and SB performed the statistical analysis and wrote the draft. CW, NS, and UL commented on the second draft. All authors commented on the final manuscript, which was completed by DN, NK, AK, SB, CW, NS, and UL.

\section{FUNDING}

This study was supported by the Thomi Hopf Stiftung, Oberwilerstrasse 65, 4123 Allschwil, Switzerland (www.thomihopf-stiftung.ch).

\section{ACKNOWLEDGMENTS}

The authors thank Marielle Koenig (UPK) and Vladimir Djurdjevic (UPK) for the much appreciated support and planning. Further, we thank Vivien Ankli (University of Basel) for helping us in data gathering and entry. Finally, we thank Claudia Züger (University of Basel) for proofreading as well as to all who have contributed to this project.

suffering from major depressive disorder. J Psychiatr Res (2016) 76:1-8. doi: 10.1016/j.jpsychires.2016.01.012

12. Cuijpers P, Berking M, Andersson G, Quigley L, Kleiboer A, Dobson KS. A meta-analysis of cognitive-behavioural therapy for adult depression, alone and in comparison with other treatments. Can J Psychiatry (2013) 58:376-85. doi: $10.1177 / 070674371305800702$

13. Li JM, Zhang Y, Su WJ, Liu LL, Gong H, Peng W, et al. Cognitive behavioral therapy for treatment-resistant depression: A systematic review and metaanalysis. Psychiatry Res (2018) 268:243-50. doi: 10.1016/j.psychres. 2018.07.020

14. Schuch FB, Vasconcelos-Moreno MP, Borowsky C, Zimmermann AB, Rocha NS, Fleck MP. Exercise and severe major depression: effect on symptom severity and quality of life at discharge in an inpatient cohort. J Psychiatr Res (2015) 61:25-32. doi: 10.1016/j.jpsychires.2014.11.005

15. Schuch FB, Morres ID, Ekkekakis P, Rosenbaum S, Stubbs B. A critical review of exercise as a treatment for clinically depressed adults: time to get pragmatic. Acta Neuropsychiatr (2016) 29(2):1-7. doi: 10.1017/neu.2016.21

16. Stubbs B, Rosenbaum S, Vancampfort D, Ward PB, Schuch FB. Exercise improves cardiorespiratory fitness in people with depression: A meta-analysis of randomized control trials. J Affect Disord (2016) 190:249-53. doi: 10.1016/ j.jad.2015.10.010

17. Schuch F, Vancampfort D, Firth J, Rosenbaum S, Ward P, Reichert T, et al. Physical activity and sedentary behavior in people with major depressive disorder: A systematic review and meta-analysis. J Affect Disord (2017) 210:139-50. doi: 10.1016/j.jad.2016.10.050

18. Stansfeld S, Candy B. Psychosocial work environment and mental health-a meta-analytic review. Scand J Work Environ Health (2006) 32:443-62. doi: 10.5271/sjweh.1050

19. Netterstrøm B, Conrad N, Bech P, Fink P, Olsen O, Rugulies R, et al. The Relation between Work-related Psychosocial Factors and the Development of Depression. Epidemiologic Rev (2008) 30:118-32. doi: 10.1093/epirev/ mxn004

20. Nieuwenhuijsen K, Bruinvels D, Frings-Dresen M. Psychosocial work environment and stress-related disorders, a systematic review. Occup Med (2010) 60:277-86. doi: 10.1093/occmed/kqq081

21. Ndjaboué R, Brisson C, Vézina M. Organisational justice and mental health: a systematic review of prospective studies. Occup Environ Med (2012) 69:694700. doi: 10.1136/oemed-2011-100595

22. Virtanen M, Nyberg ST, Batty GD, Jokela M, Heikkilä K, Fransson EI, et al. Perceived job insecurity as a risk factor for incident coronary heart disease: 
systematic review and meta-analysis. BMJ : Br Med J (2013) 347:f4746. doi: 10.1136/bmj.f4746

23. Stuke H, Bermpohl F. Welche Arbeitsbedingungen begünstigen die Entwicklung einer depressiven Störung? Psychiat Prax (2016) 43:245-52. doi: 10.1055/s-0035-1552769

24. Lerner D, Adler DA, Chang H, Lapitsky L, Hood MY, Perissinotto C, et al. Unemployment, job retention, and productivity loss among employees with depression. Psychiatr Serv (2004) 55:1371-8. doi: 10.1176/appi.ps.55.12.1371

25. Lépine J-P, Briley M. The increasing burden of depression. Neuropsychiatr Dis Treat (2011) 7:3. doi: 10.2147/NDT.S19617

26. Evans-Lacko S, Koeser L, Knapp M, Longhitano C, Zohar J, Kuhn K. Evaluating the economic impact of screening and treatment for depression in the workplace. Eur Neuropsychopharmacol (2016) 26:1004-13. doi: 10.1016/j.euroneuro.2016.03.005

27. Amos TB, Tandon N, Lefebvre P, Pilon D, Kamstra RL, Pivneva I, et al. Direct and Indirect Cost Burden and Change of Employment Status in TreatmentResistant Depression: A Matched-Cohort Study Using a US Commercial Claims Database. J Clin Psychiatry (2018) 79:1-9. doi: 10.4088/JCP.17m11725

28. Stewart WF, Ricci JA, Chee E, Hahn SR, Morganstein D. Cost of Lost Productive Work Time Among US Workers With Depression. JAMA (2003) 289:3135-44. doi: 10.1001/jama.289.23.3135

29. Mcternan WP, Dollard MF, Lamontagne AD. Depression in the workplace: An economic cost analysis of depression-related productivity loss attributable to job strain and bullying. Work Stress (2013) 27:321-38. doi: 10.1080/ 02678373.2013.846948

30. Mccrone P, Rost F, Koeser L, Koutoufa I, Stephanou S, Knapp M, et al. The economic cost of treatment-resistant depression in patients referred to a specialist service. J Ment Health (2018) 27:567-73. doi: 10.1080/ 09638237.2017.1417562

31. Cuijpers P, Van Straten A, Hollon SD, Andersson G. The contribution of active medication to combined treatments of psychotherapy and pharmacotherapy for adult depression: a meta-analysis. Acta Psychiatrica Scandinavica (2010) 121:415-23. doi: 10.1111/j.1600-0447.2009.01513.x

32. Schramm E, Berger M. [Interpersonal psychotherapy for work-related stress depressive disorders]. Nervenarzt (2013) 84:813-22. doi: 10.1007/s00115-0133744-5

33. Knekt P, Lindfors $\mathrm{O}$, Laaksonen MA, Raitasalo R, Haaramo P, Järvikoski A. Effectiveness of short-term and long-term psychotherapy on work ability and functional capacity - A randomized clinical trial on depressive and anxiety disorders. J Affect Disord (2008) 107:95-106. doi: 10.1016/j.jad.2007.08.005

34. Hange D, Ariai N, Kivi M, Eriksson MC, Nejati S, Petersson E-L. The impact of internet-based cognitive behavior therapy on work ability in patients with depression-a randomized controlled study. Int J Gen Med (2017) 10:151. doi: 10.2147/IJGM.S129710

35. Hallgren M, Kraepelien M, Lindefors N, Zeebari Z, Kaldo V, Forsell Y. Physical exercise and internet-based cognitive-behavioural therapy in the treatment of depression: randomised controlled trial. Br J Psychiatry (2015) 207:227-34. doi: 10.1192/bjp.bp.114.160101

36. Johnson JE, Price AB, Kao JC, Fernandes K, Stout R, Gobin RL, et al. Interpersonal psychotherapy (IPT) for major depression following perinatal loss: a pilot randomized controlled trial. Arch Women's Ment Health (2016) 19:845-59. doi: 10.1007/s00737-016-0625-5

37. Lemmens LH, Galindo-Garre F, Arntz A, Peeters F, Hollon SD, Derubeis RJ, et al. Exploring mechanisms of change in cognitive therapy and interpersonal psychotherapy for adult depression. Behav Res Ther (2017) 94:81-92. doi: 10.1016/j.brat.2017.05.005

38. Santor DA, Kusumakar V. Open Trial of Interpersonal Therapy in Adolescents With Moderate to Severe Major Depression: Effectiveness of Novice IPT Therapists. J Am Acad Child Adolesc Psychiatry (2001) 40:23640. doi: 10.1097/00004583-200102000-00019

39. Göder R, Fritzer G, Hinze-Selch D, Huchzermeier C, Koch JM, Seeck-Hirschner $\mathrm{M}$, et al. Sleep in Major Depression: Relation to Memory Performance and Outcome after Interpersonal Psychotherapy. Neuropsychobiology (2007) 55:36-42. doi: 10.1159/000103574

40. Dombrovski AY, Cyranowski JM, Mulsant BH, Houck PR, Buysse DJ, Andreescu C, et al. Which symptoms predict recurrence of depression in women treated with maintenance interpersonal psychotherapy? Depression Anxiety (2008) 25:1060-6. doi: 10.1002/da.20467

41. World Medical Association. World Medical Association Declaration of Helsinki: ethical principles for medical research involving human subjects. JAMA (2013) 310:2191-4. doi: 10.1001/jama.2013.281053

42. Saß H, Wittchen H-U, Zaudig M. (1996). Diagnostisches und statistisches Manual psychischer Störungen-DSM-IV. Deutsche Bearbeitung u. Einleitung von H. Sass, H.-U. Wittchen, M. Zaudig.

43. Wittchen H-U, Zaudig M, Fydrich T. Skid. Strukturiertes klinisches Interview für DSM-IV. (1997). Göttingen: Hogrefe.

44. Hamilton M. A rating scale for depression. J Neurology Neurosurgery Psychiatry (1960) 23:56-61. doi: 10.1037/t04100-000

45. Beck AT, Steer RA, Brown GK, Hautzinger M, Keller F, Kühner C. Beck Depressionsinventar, 2. Auflage (BDI-II). Frankfurt am Main: Pearson Assessment (2006).

46. Beck AT, Steer RA, Garbin GM. Psychometric properties of the Beck Depression Inventory: Twenty-five years of evaluation. Clin Psychol Rev (1988) 8:77-100. doi: 10.1016/0272-7358(88)90050-5

47. WAI-Manual [Online]. (2015). Available: http://www.arbeitsfaehigkeit.uniwuppertal.de/picture/upload/file/WAI-Manual.pdf [Accessed].

48. Lagerveld SE, Blonk RW, Brenninkmeijer V, Wijngaards-De Meij L, Schaufeli WB. Work-focused treatment of common mental disorders and return to work: a comparative outcome study. J Occup Health Psychol (2012) 17:220-34. doi: $10.1037 / \mathrm{a} 0027049$

49. Morin CM, Belleville G, Bélanger L, Ivers H. The Insomnia Severity Index: psychometric indicators to detect insomnia cases and evaluate treatment response. Sleep (2011) 34:601-8. doi: 10.1093/sleep/34.5.601

50. Gerber M, Lang C, Lemola S, Colledge F, Kalak N, Holsboer-Trachsler E, et al. Validation of the German version of the insomnia severity index in adolescents, young adults and adult workers: results from three crosssectional studies. BMC Psychiatry (2016) 16:174. doi: 10.1186/s12888-0160876-8

51. Schramm E, Mack S. W-IPT Guideline (in prep).

52. Julious SA. Sample size of 12 per group rule of thumb for a pilot study. Pharmaceutical Stat (2005) 4:287-91. doi: 10.1002/pst.185

53. Becker BJ. Synthesizing standardized mean-change measures. Br J Math Stat Psychol (1988) 41:257-78. doi: 10.1111/j.2044-8317.1988.tb00901.x

54. Cohen J. A power primer. psychol Bull (1992) 112:155. doi: 10.1037/00332909.112.1.155

55. Klerman G, Weissman M, Rounsaville B, Chevron E. "Interpersonal therapy of depression (IPT)". New York: Basic Books (1984).

56. Wasserstein RL, Schirm AL, Lazar NA. "Moving to a world beyond " $p<0.05$ "”. (2019). The American Statistician. doi: 10.1080/00031305.2019.1583913

57. Evans-Lacko S, Knapp M. Global patterns of workplace productivity for people with depression: absenteeism and presenteeism costs across eight diverse countries. Soc Psychiatry Psychiatr Epidemiol (2016) 51:1525-37. doi: 10.1007/s00127-016-1278-4

58. Brand S, Gerber M, Hatzinger M, Beck J, Holsboer-Trachsler E. Evidence for similarities between adolescents and parents in sleep patterns. Sleep Med (2009) 10:1124-31. doi: 10.1016/j.sleep.2008.12.013

59. Bajoghli H, Alipouri A, Holsboer-Trachsler E, Brand S. Sleep patterns and psychological functioning in families in northeastern Iran; evidence for similarities between adolescent children and their parents. $J$ adolescence (2013) 36:1103-13. doi: 10.1016/j.adolescence.2013.08.016

Conflict of Interest: The authors declare that the research was conducted in the absence of any commercial or financial relationships that could be construed as a potential conflict of interest.

Copyright (c) 2020 Niedermoser, Kalak, Kiyhankhadiv, Brand, Walter, Schweinfurth and Lang. This is an open-access article distributed under the terms of the Creative Commons Attribution License (CC BY). The use, distribution or reproduction in other forums is permitted, provided the original author(s) and the copyright owner(s) are credited and that the original publication in this journal is cited, in accordance with accepted academic practice. No use, distribution or reproduction is permitted which does not comply with these terms. 